\title{
Differences in the expression of cell envelope proteinases (CEP) in two Lactobacillus paracasei probiotic strains
}

\author{
José María Coll-Marqués, Christine Bäuerl, Manuel Zúñiga and \\ Gaspar Pérez-Martínez ${ }^{*} \dagger$
}

Department of Food Biotechnology, Instituto de Agroquímica y Tecnología de Alimentos, Spanish National Research Council (CSIC), Agustín Escardino Benlloch, 7, 46980 Paterna, Valencia, Spain

*Corresponding author: Instituto de Agroquímica y Tecnología de Alimentos, Consejo Superior de Investigaciones Científicas (Spanish National Research Council) (CSIC), Av. Agustín Escardino Benlloch, 7, 46980 Valencia, Spain. Tel: +34 9639000 22; E-mail: gaspar.perez@iata.csic.es

One sentence summary: This work dissects the molecular regulation of the cell wall proteinase and related genes in two probiotic strains of Lactobacillus paracasei.

Editor: Akihito Endo

${ }^{\dagger}$ Gaspar Pérez-Martínez, http://orcid.org/0000-0003-3501-1626

\begin{abstract}
Proteinase PrtP (EC:3.4.21.96) is a cell envelope proteinase (CEP) highly expressed in the probiotic strain Lactobacillus paracasei BL312(VSL\#3) that accounts for its anti-inflammatory properties. The main aim of this work is to understand differences in CEP expression between this strain and L. paracasei BL23. Hence, differences in the regulation by amino acid sources of four proteinase related genes (prtP, prsA, prtR1 and prtR2) were determined by RT-qPCR in BL312(VSL\#3) and BL23 using as a reference BL368, a BL23 derepressed mutant lacking the response regulator (RR) PrcR. BL312(VSL\#3) showed greater expression of prtP (2- to 3-fold) than BL23, and prtP was highly repressed by peptone in both strains. Two other putative CEP genes, prtR1 and prtR2, showed a low expression profile. Interestingly, when the prsA-prtP promoter region from both strains, and deleted mutants, were cloned in vector PT1GR, expression of the gfp and mrfp fluorescent reporters was always repressed in BL23 (high or low peptone) and derepressed in BL368, revealing an interesting mechanism of regulation affecting specifically to this promoter. In conclusion, BL312(VSL\#3) has higher expression of prtP and other CEP related genes than BL23, that could respond to a natural deregulation in this strain, possibly independent from the RR PrcR.
\end{abstract}

Keywords: Proteinases; probiotic; Lactobacillus paracasei; regulation features; expression vector; RT-qPCR

\section{INTRODUCTION}

Cell envelope proteinases (CEP) from lactic acid bacteria (LAB), generically called lactocepins, constitute a family of unusually large proteins that belong to the group of cell wall-anchored subtilisin-like PII-type proteinases (EC:3.4.21.96). In Lactococcus lactis and closely related species, CEP was designated PrtP and it was studied for their relevance in cheese production, as it plays an important role in the physicochemical and sensorial evolution of cheese and other dairy products (Law and Haandrikman 1997; Broadbent et al. 1998, 2002; Chen et al. 2017). In the last decade, these proteinases have been highlighted by their potential functional activity, for instance generating peptides that inhibit the angiotensin-converting enzyme (ACE, reviewed by Raveschot et al. (2018)). They have also been related to the potential reduction of milk allergies by destroying allergens of $\beta$-lactoglobulin (Pescuma et al. 2015) and $\alpha$ S1-caseins 
(El-Ghaish et al. 2011). Furthermore, lactocepin from the Lactobacillus paracasei strain found in the commercial probiotic mixture VSL\#3 selectively degraded IP-10, resulting in a significantly reduced inflammatory profile and lymphocyte recruitment after intraperitoneal injection in a murine ileitis model (von Schillde et al. 2012). In fact, these activities could be the mechanisms underlying the observed anti-allergic and anti-inflammatory effects of dairy products containing lactobacilli (Lee et al. 2007; Gobbato, Rachid and Perdigon 2008; Chaves, Perdigon and de LeBlanc 2011; Sheikhi et al. 2012). For this reason, knowledge on factors that regulate this enzymatic activity is essential for the selection of proteinase proficient strains and the improvement of probiotic lactobacilli performance, particularly in the $L$. casei/paracasei taxon.

In L. paracasei BL23, the genes encoding PrtP (prtP, LCABL_24 520) and its maturase PrtM (prsA, LCABL_24 530) are adjacent and transcribed in opposite direction, hence the promoters for both genes are in the prsA-prtP intergenic region. When this promoter region was cloned upstream the $\beta$-glucuronosidase gene (gusA) reporter gene a repressing effect was found on a peptide rich medium (containing casitone) on the gene expression of prtP and prsA (Pastar et al. 2007). A more recent study described that the promoter region of prtP and prsA is recognized by the response regulator (RR) PrcR. PrcR acts as a repressor of the promoter activity on peptide rich growth media and, interestingly, it binds to the promoter region of those genes even in the absence of the canonical phosphate supplier, acetyl-P (Alcantara et al. 2016).

Furthermore, five subgroups of CEPs have been described in LAB according to their domain composition: PrtB from Lactobacillus delbrueckii subsp. bulgaricus, PrtH from Lactobacillus helveticus, PrtP from Lc. lactis and Lactobacillus paracasei, PrtR from Lactobacillus rhamnosus and PrtS from Streptococcus thermophilus (Siezen 1999; Fernandez-Espla et al. 2000; Pastar et al. 2003; Savijoki, Ingmer and Varmanen 2006; Alcantara et al. 2016). After detailed analysis of L. paracasei BL23 genome a cluster of two proteinase genes was found that encoded proteins homologous to PrtR from Lactobacillus rhamnosus (Pastar et al. 2003; Muñoz-Provencio et al. 2012), prtR1 (LCALB-0 5520) and prtR2 (LCALB 0 5530). However, little is known about their function or expression in L. paracasei.

This work explored the regulatory effect of amino acids on prtP and prsA genes in the probiotic and laboratory strain L. paracasei BL23, using a highly proteolytic deregulated mutant (BL368) and a commercial probiotic isolate (L. paracasei VSL\#3). For this purpose, we used RT-qPCR and designed a new expression vector carrying the genes encoding fluorescent reporters GFPuv and RFP.

\section{MATERIALS AND METHODS}

\section{Bacterial strains and culture conditions}

Strain L. paracasei BL23 is a laboratory strain (Acedo-Felix and Perez-Martinez 2003) and L. paracasei BL368 is a derivative mutant of BL23 in the RR PrcR that displayed faster milk clotting and acidification properties (Alcantara et al. 2016). L. paracasei from the probiotic mixture VSL\#3 (here BL312) was a generous gift from Dr. Claudio De Simone (L'Aquila, Italy). All lactobacilli were grown under static conditions in MRS medium (Difco) at $37^{\circ} \mathrm{C}$. L. paracasei strains carrying expression vectors were grown on MRS broth or agar with $5 \mu \mathrm{g} / \mathrm{mL}$ erythromycin. For the assays with high $(20 \mathrm{~g} / \mathrm{L})$ and low $(1 \mathrm{~g} / \mathrm{L})$ peptone as main source of peptides and amino acids, strains were grown on a semi-defined medium (SDM) modified from Morishita et al. (1981), from which all sources of amino acids were eliminated except yeast extract. The rest of the components were identical to the original recipe containing per litre: 5 g yeast extract (Difco Laboratories, Detroit, MI, USA), 5 g D-glucose, 1.7 g sodium acetate, $2 \mathrm{~g}$ ammonium citrate, $3 \mathrm{~g} \mathrm{KH}_{2} \mathrm{PO}_{4}, 3 \mathrm{~g} \mathrm{~K}_{2} \mathrm{HPO}_{4}, 0.575 \mathrm{~g} \mathrm{MgSO}_{4} .7 \mathrm{H}_{2} \mathrm{O}, 0.034 \mathrm{~g}$ $\mathrm{FeSO}_{4} .7 \mathrm{H}_{2} \mathrm{O}, 0.12 \mathrm{~g} \mathrm{MnSO}_{4} .2 \mathrm{H}_{2} \mathrm{O}, 0.5 \mathrm{~g}$ L-cysteine hydrochloride and $1 \mathrm{~g}$ Tween 80; final $\mathrm{pH}$ was adjusted to 7.2. E. coli DH10B and $\mathrm{DH} 5 \alpha$ were the intermediate cloning hosts, and they were grown under aerobic conditions at $37^{\circ} \mathrm{C}$ in LB Broth (Difco) or LB with $2 \%$ agar containing $300 \mu \mathrm{g} / \mathrm{mL}$ erythromycin. In case of pT1Nx derivatives, Lactococcus lactis subsp. cremoris MG1363 was used as intermediate host to transform vector ligations. Plasmid carrying strains were grown on M17 (Difco) with 5 g/l glucose (GM17) liquid or with $1.8 \%$ agar and $5 \mu \mathrm{g} / \mathrm{mL}$ erythromycin.

\section{DNA manipulation and cloning}

The close phylogenetic relationship between strain BL312(VSL\#3) and BL23 allowed designing oligonucleotides to sequence prtP and prsA genes in order to obtain the complete sequence of the prtP-prsA genes and intergenic region by chromosome walking. The primers used and the complete sequence are described in Table S1 (Supporting Information).

The reporter genes gfp and mrfp from vector pTLGR (GarcíaCayuela et al. 2012), that express respectively green fluorescent and red fluorescent proteins, were subcloned. Specific details on restriction enzymes used, blunt end generation with Klenow enzyme or cloning by PCR amplification are described in Fig. 1. Briefly, first the expression cassette $g f p$ - $m r f p$ was subcloned in the BamHI/SmaI sites of vector pIAV7 (Perez-Arellano et al. 2003) made blunt with Klenow enzyme yielding PIA-GR, which would facilitate transformation in E. coli and a stable maintenance in lactobacilli. All promoter versions - including deleted mutants were PCR amplified and ligated to BamHI-digested PIA-GR, and the whole cassette - promoter and reporter genes - were then subcloned in the EcoRI-Klenow blunt/SpeI sites of the expression vector pT1NX (Steidler et al. 2000). Plasmid pT1GR is identical to PT1GR::PprsA-PprtP but with no promoter sequences between the reporter genes, and it was obtained by direct amplification of the gfp-mrfp cassette from pIA-GR, that was then cloned in pT1NX (EcoRI-Klenow blunt/SpeI).

\section{Expression analysis of fluorescent reporters}

The expression of the reporter genes was determined in 96well plates during $12 \mathrm{~h}$ incubation in the corresponding medium inoculated with the different strains at least in triplicate using a POLARstar plate reader instrument (BMG Labtech, Offenburg, Germany) equipped with a temperature-controlled plate and shaker. GFP fluorescence was determined with excitation/emission filters of $485 / 520 \mathrm{~nm}$ and the Cherry mRFP fluorescence was measured using excitation/emission filters of $540 / 612 \mathrm{~nm}$. Automatic recording of data was set up every $5 \mathrm{~min}$ and fluorescence was determined for all strains and conditions during $12 \mathrm{~h}$ (720 min); if maximum was reached before $12 \mathrm{~h}$ this was the value selected for graphical representation of relative fluorescence. Differences were calculated relative to the control vector (pT1-TGR) bearing both fluorescence genes but lacking any promotor sequence. Fluorescence values were corrected for growth (absorbance at $590 \mathrm{~nm}$ ) at the corresponding time points. A general linear model was applied for the analysis of variance (ANOVA) individually of green and red fluorescence data in the two strains BL23 and BL368, using IBM SPSS package software. Significance level was calculated according to HSD Tukey test. 

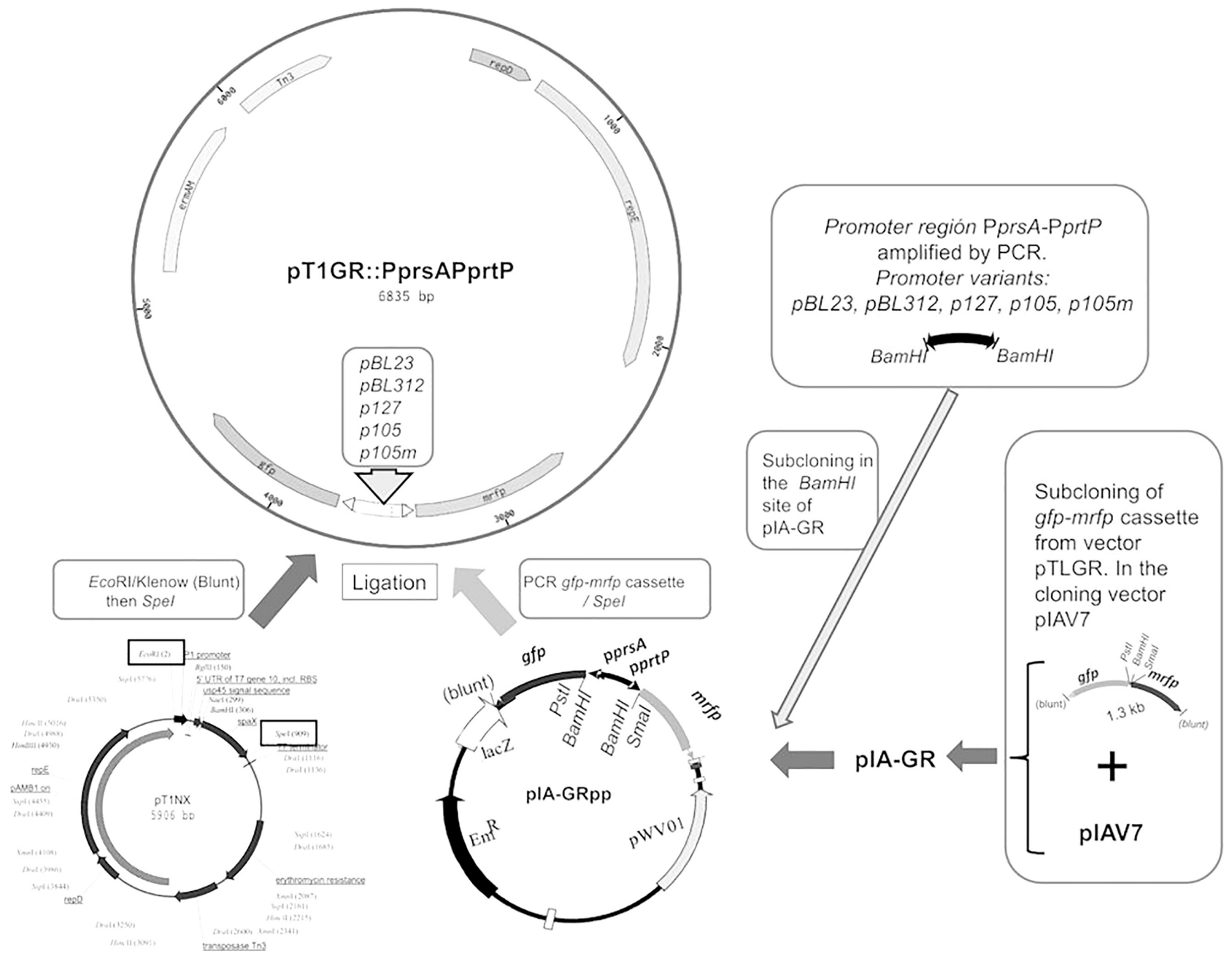

Figure 1. Summarized diagram of the steps followed for the construction of the reporter plasmids used in this work. For a more detailed description check Figure S1 (Supporting Information).

\section{RNA isolation and RT-qPCR}

RNA extraction and the general procedure for RT-qPCR was as described before (Landete et al. 2010). Briefly, RNA isolation was performed using the TRIzol reagent (Invitrogen, Carlsbad, CA, USA) and RNA integrity was confirmed using the Experion automated electrophoresis system (Bio-Rad, Hercules, CA, USA). Contaminating genomic DNA was digested using DNaseI (Ref. AMP-D1, Sigma, St. Louis, MO, USA) and cDNA synthesis was performed using the Transcriptor First Strand cDNA Synthesis Kit (Roche Diagnostics, Mannheim, Germany) according to the instructions of the manufacturer. A total of four housekeeping genes, iles, lepA, pcrA and pyrG, were tested to normalize gene expression, that were respectively encoding isoleucyl-tRNA synthetase, GTP-binding protein, ATP-dependent DNA helicase and CTP synthetase. The primer sequences for PrtR1 and PrtR2 were designed using the Primer Blast tool (https://www.ncbi.n lm.nih.gov/tools/primer-blast/) and are listed in Table 2. Gene expression ratios were calculated using the software tool REST based on the efficiency-corrected method (Pfaffl, Horgan and Dempfle 2002). When bacterial strains were grown under different peptone concentrations, lepA and pyrG showed no stable gene expression for which they were disregarded in their respective calculations.

\section{RESULTS}

Transcription analysis of CEP related genes in $L$. paracasei strains

The repressing effect by rich amino acid sources on proteinase activity has already been studied in L. paracasei (Pastar et al. 2007; Alcantara et al. 2016); however, differences between probiotic strains at transcriptional level had not been described, as it is the case of L. paracasei BL23 and L. paracasei BL312(VSL\#3). For this purpose, expression of the chromosomal genes encoding CEP related genes (prtP, prsA, prtR1 and prtR2) was analysed by RT-qPCR. In addition to prtP and prsA, encoding PrtP and the associated maturase PrtM, two new CEP paralogous genes found in the genome of BL23 were included. They showed great similarity to L. rhamnosus prtR, prtR1 (LCABL_0 5330) and prtR2 (LCABL_0 5320) (Muñoz-Provencio et al. 2012) and similar counterparts were present in BL312 (VSL\#3), as deduced by the amplification with specific qPCR primers. Differential expression of all CEP related genes (prtP, prsA, prtR1 and prtR2) was tested on 1 and $20 \mathrm{~g} / \mathrm{L}$ peptone (Table 1 ). Since SDM is a poor medium, $1 \mathrm{~g} / \mathrm{L}$ peptone was required to support growth of BL23, and it did not activate PrcR dependent repression, as shown by the results. In strains BL312(VSL\#3) and BL23 expression of 
Table 1. Relative gene expression ratios of the of genes prtP, prsA, prtR1 and prtR2 in strains BL23, BL312(VSL\#3) and BL368 grown with different peptone concentrations. The upper part of the table shows intra-strain comparisons of gene expression due to peptone concentration. The lower part shows gene expression between strains relative to strain BL23.

\begin{tabular}{|c|c|c|c|c|c|c|c|c|c|}
\hline \multirow[b]{2}{*}{ Peptone } & \multirow[b]{2}{*}{ Strain } & \multicolumn{2}{|c|}{ prtP } & \multicolumn{2}{|c|}{ prsA } & \multicolumn{2}{|c|}{ prtR1 } & \multicolumn{2}{|c|}{ prtR2 } \\
\hline & & Exp. rate & Std. Err range & Exp. rate & $\begin{array}{l}\text { Std. Error } \\
\text { range }\end{array}$ & Exp. rate & $\begin{array}{l}\text { Std. Error } \\
\text { range }\end{array}$ & Exp. rate & $\begin{array}{l}\text { Std. Error } \\
\text { range }\end{array}$ \\
\hline $1 \mathrm{~g} / \mathrm{L}$ & BL23 & 1 & & 1 & & 1 & & 1 & \\
\hline $20 \mathrm{~g} / \mathrm{L}$ & & $0.178^{*}$ & $0.173-0.183$ & 0.937 & $0.872-1.006$ & 0.721 & $0.688-0.756$ & 0.888 & 0.777-1.019 \\
\hline $1 \mathrm{~g} / \mathrm{L}$ & BL312 & 1 & & 1 & & 1 & & 1 & \\
\hline $20 \mathrm{~g} / \mathrm{L}$ & & $0.313^{*}$ & $0.299-0.328$ & $0.738^{*}$ & $0.709-0.768$ & $0.688^{*}$ & $0.666-0.712$ & $0.854^{*}$ & $0.795-0.917$ \\
\hline $1 \mathrm{~g} / \mathrm{L}$ & BL368 & 1 & & 1 & & 1 & & 1 & \\
\hline $20 \mathrm{~g} / \mathrm{L}$ & & 1.054 & $1.026-1.082$ & 2.058 & $1.992-2.128$ & $0.809^{*}$ & $0.790-0.829$ & $1.021^{*}$ & $1.013-1.029$ \\
\hline \multirow[t]{3}{*}{$1 \mathrm{~g} / \mathrm{L}$} & BL23 & 1 & & 1 & & 1 & & 1 & \\
\hline & BL312 & 2.008 & $1.915-2.107$ & 2.674 & $2.486-2.880$ & $6.695^{*}$ & $6.380-7.034$ & $57.467^{*}$ & $50.269-66.023$ \\
\hline & BL368 & $9.434^{*}$ & $9.180-9.697$ & 1.093 & $1.017-1.175$ & 0.966 & $0.921-1.013$ & 1.539 & $1.347-1.759$ \\
\hline \multirow[t]{3}{*}{$20 \mathrm{~g} / \mathrm{L}$} & BL23 & 1 & & 1 & & 1 & & 1 & \\
\hline & BL312 & 3.523 & $3.470-3.577$ & $2.106^{*}$ & $2.092-2.120$ & $6.390^{*}$ & $6.366-6.413$ & $55.217^{*}$ & $52.500-58.082$ \\
\hline & BL368 & $55.767^{*}$ & $54.271-57.317$ & 2.401 & $2.325-2.480$ & 1.084 & $1.059-1.109$ & $1.769^{*}$ & $1.682-1.861$ \\
\hline
\end{tabular}

(*) $P$ value $\leq 0.05$

Table 2. Primers used in this study.

\begin{tabular}{llcc}
\hline Primer name & \multicolumn{1}{c}{ Sequence 5'-3' } & Features & Ref. \\
\hline Pro1 & GGCGAATTCCAAGCAAAGGCGGC & EcoRI & This work \\
Pro2 & CATCGAGCTCGCCTTAGCATCAG & SacI & This work \\
Prom_prt_F & AATTGGATCCTGAAATGCTTACAGTAAACG & BamHI & This work \\
Prom_prt_R & & BamHI- SmaI & This work \\
& AATTGGATCCATAACCCGGGTTCCCAAAAGTTTCAGAACC & & This work \\
PprtP_cor & CGGGATCCAAGATTAAAATTTTCGTTGAATCTGT & BamHI & This work \\
PprtP_corm & CGGGATCCTTGAATGTAATATACGTTGAATCTG & BamHI, & Pastar et al. (2007) \\
M94F & CGGAATTCCGCGGTCTCCTCTGAAATGCTT & BamHI & Pastar et al. (2007) \\
M94R & CGGGATCCCGCATTATTAGAAAATGAGTATTTGC & PamHI & Pastar et al. (2007) \\
P127F & CGGGATCCCGCAGATAGGAAAATTAATAAAAGATTAA & BamHI & This work \\
P127R & CGGAATTCCGCCAATACCCTCCACTTCCC & This work \\
TLGR_compl_F & TAGTCGACTAGTCTCGAGCCTATTTGTATAGTTCATCC & EcoRI & SalI-SpeI-XhoI \\
TLGR_compl_R & GCTTCTCAAGGGCATCGGTCG & & Alcantara et al. (2016) \\
PrtP for & CTTCTGAACGCTGGCATC & RT_qPCR & Alcantara et al. (2016) \\
PrtP rev & GCCATTATCTGATGAACAGG & RT_qPCR & Alcantara et al. (2016) \\
PrtM for & GCCCAAAGTGACTGTCCAGC & RT_qPCR & Alcantara et al. (2016) \\
PrtM rev & TTCGCAAGCGTGGCAAAATC & RT_qPCR & This work \\
PrtR1 for & AAACACCAGCGTCACCAGTC & RT_qPCR & This work \\
PrtR1 rev & GCGGTTGCACATTCCCTTTG & RT_qPCR & This work \\
PrtR2 for & ATGGTGCCTGCAAAGGATGG & RT_qPCR & This work \\
PrtR2 rev & GGCCACTGGTAAGCTCATGG & RT_qPCR &
\end{tabular}

prtP was remarkably repressed and all other genes were moderately affected by amino acids. As expected, peptone did not affect expression of prtP in the deregulated mutant BL368. Under conditions of low amino acid supply, the greatest expression ratios corresponded to prtP in BL368 and prtR1 and prtR2 in BL312(VSL\#3). At the highest peptone concentration, prtP gene expression in strain BL312(VSL\#3) decreased but remained proportionally higher than in BL23, thus indicating that the higher activity/expression of prtP in BL312(VSL\#3) may be related to other strain specific factors, not to a deregulation by nitrogen sources. On the other hand, BL368 showed a much greater expression of prtP but not of the other genes, indicating that they must be moderately regulated by PrcR, as previously reported (Alcantara et al. 2016).
The relatively higher expression of prtR1 and prtR2 in BL312(VSL\#3) could be interpreted as if these genes were highly expressed; however, the careful observation of RT-qPCR data showed that prtR1 and prtR2 normally appeared at high Cts. Since prsA showed relatively uniform expression, it was considered a suitable internal reference to compare differences in the expression of all the genes within each strain (Table S2, Supporting Information) the greatest differences were detected on $1 \mathrm{~g} / \mathrm{L}$ peptone, where prtP expression was 7- to 10-fold greater than prsA in strains BL23 and BL312 and, in BL368, prtP reached 83.8-fold the expression of prsA, suggesting that prtP suffered a much tighter control by PrcR, than prsA. Also, these numbers showed a poor relative expression of prtR1 and prtR2. Only in strain BL312 grown on high amino acid concentration, expres- 
(A)
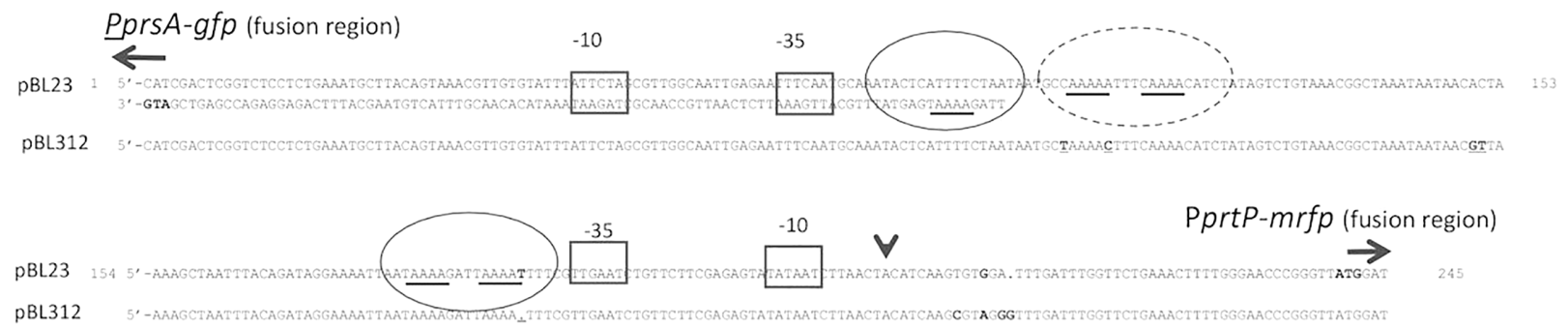

p127

p105

p105m

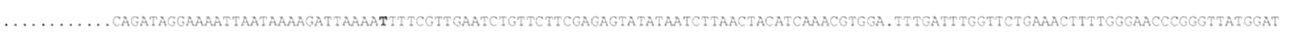

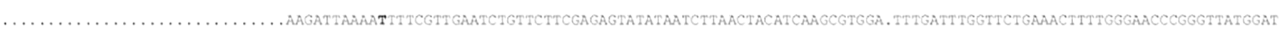

.........................ttagatgt ȦATAT aCGTT GARTCTGTTCTTCGAGAGTATATAATCTTAACTACATCAAGCGTGGA. TTTGATT TGGTTCTGAAACTTTTGGGAACCCGGGTTATGGAT

(B)

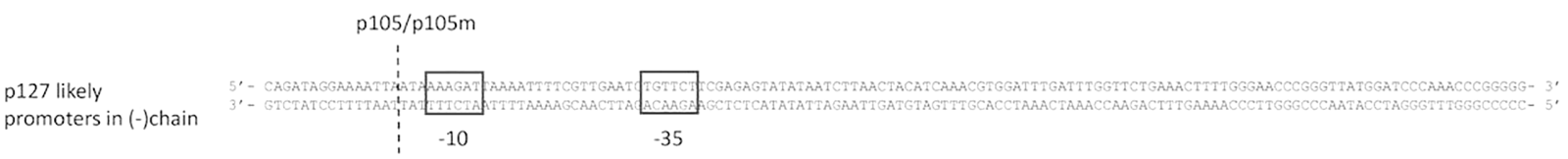

Figure 2. Nucleotide sequence of the prsA-prtP intergenic region cloned in the expression vector pT1GR, that contains the promoter regions (-10 and -35) framed in square boxes. Panel A shows the sequences of the promoters tested in the reporter vector with sequence differences highlighted in bold, where the fusion regions with the reporter genes are indicated by horizontal arrows, the transcriptional start point is shown by a vertical arrow and the possible PrcR dependent regulatory regions are underlined and surrounded by ellipses. In pBL23, an uncharacterized regulatory region of prtP is shown in the (+)chain surrounded by a dotted ellipse. Panel B shows the putative promoter region found in the (-)chain that would explain the expression of gfp in the deleted promoter versions.

sion of prtR2 was comparable to prtP (prtR2 2.2 and prtP 3.2-fold, relative to prsA).

Cloning and mutation of the prsA-prtP intergenic region containing the divergent promoters

Since prtP is the gene with highest expression in these probiotic strains, the promoter structure of the prsA-prtP intergenic region from BL23 and BL312(VSL\#3) was PCR amplified and cloned in specially designed vectors with divergent reporter genes. Reporter genes were obtained from vector pTLGR (GarcíaCayuela et al. 2012), so that the promoters could be tested by the expression of the green fluorescent protein (GFP) from the gene $g f p^{U V}$ and the red fluorescent protein (RFP) from $m r f p$. In order to improve the stability and replicative potential of the vectors in lactobacilli, the complete expression cassette was first cloned in the wide host range plasmid pIAV7, and later subcloned in vector pT1NX (Steidler et al. 2000) to optimize expression and stability (Fig. 1 and Figure S1, Supporting Information). Vector pT1GR was the new reporter without promoter, and PT1GR::PprsAPprtP was the general name for the reporter plasmid carrying the intergenic region prsA-prtP of approx. $260 \mathrm{nt}$ from BL23, here called pBL23, or BL312(VSL\#3) (pBL312) (Fig. 2). In addition, three versions of the $p B L 23$ promoter were generated by deletion in the 5 ' region lacking the putative -10 and -35 regions of prs $A$ and the PrcR regulatory sequences (AAAA), but that included the promoter region and transcriptional start of the gene prtP as previously characterised (p127) (Pastar et al. 2007) (Fig. 2). The two other constructs -promoters $p 105$ and p105m- lacked possible regulatory region overlapping the prtP promoter (Alcantara et al. 2016). All five versions of the prsA-prtP promoter (pBL23, pBL312, p127, p105 and p105m) with their known regulatory regions, promoter sites and sequence differences are detailed in Fig. 2.
Expression analysis from the fluorescent reporter genes

All five promoter versions described above were cloned in the reporter vector PT1GR, in which the expression of the gene encoding RFP ( $m r f p$ ) would be under the control of the prtP promoter (Pprtp) and GFP gene ( $g f p)$ was controlled by the prsA promoter (Pprsa). Red and green fluorescence were monitored when plasmids were transformed in BL23 and in the deregulated mutant BL368 lacking the RR PrcR. Expression of both reporters was also determined in all recombinant strains with low and high concentrations of amino acids ( $1 \mathrm{~g} / \mathrm{L}$ and $20 \mathrm{~g} / \mathrm{L}$ peptone) (Fig. 3A and B). In the wild type strain BL23, expression of RFP from the complete fragments, pBL23 and pBL312 was similar (no statistical difference), and the highest expression was found in promoters $p 127$ and $p 105$, thus indicating at least a partial release of repression in the deleted mutants. Nevertheless, differences between cells growing in medium with low or high peptone content were not significant (Fig. 3A). When reporter plasmids were introduced in the deregulated strain BL368, expression of RFP from all promoter versions was much higher than in wild type BL23 and followed a different pattern; here pBL312 induced a significantly lower expression than pBL23 and similar to deleted $p 105$ (Fig. 3A). In this case, expression from $p$ BL23 almost reached $p 127$.

In relation to the expression of GFP from the promoter of prsA (PprsA), ANOVA showed that there was no significant effect of peptone on BL23, but expression decreased significantly in BL368. Little differences could be detected between pBL23, pBL312 and p127 in BL23, but there was a pronounced strain effect as GFP expression from the promoters pBL23 and pBL312 was remarkably derepressed in BL368 (Fig. 3B). This suggested a direct regulation of PrcR on PprsA, possibly through a partial consensus AAAA identified close to the putative -35 promoter region (Fig. 2B). Surprisingly, all the deleted promoters allowed 
(A)

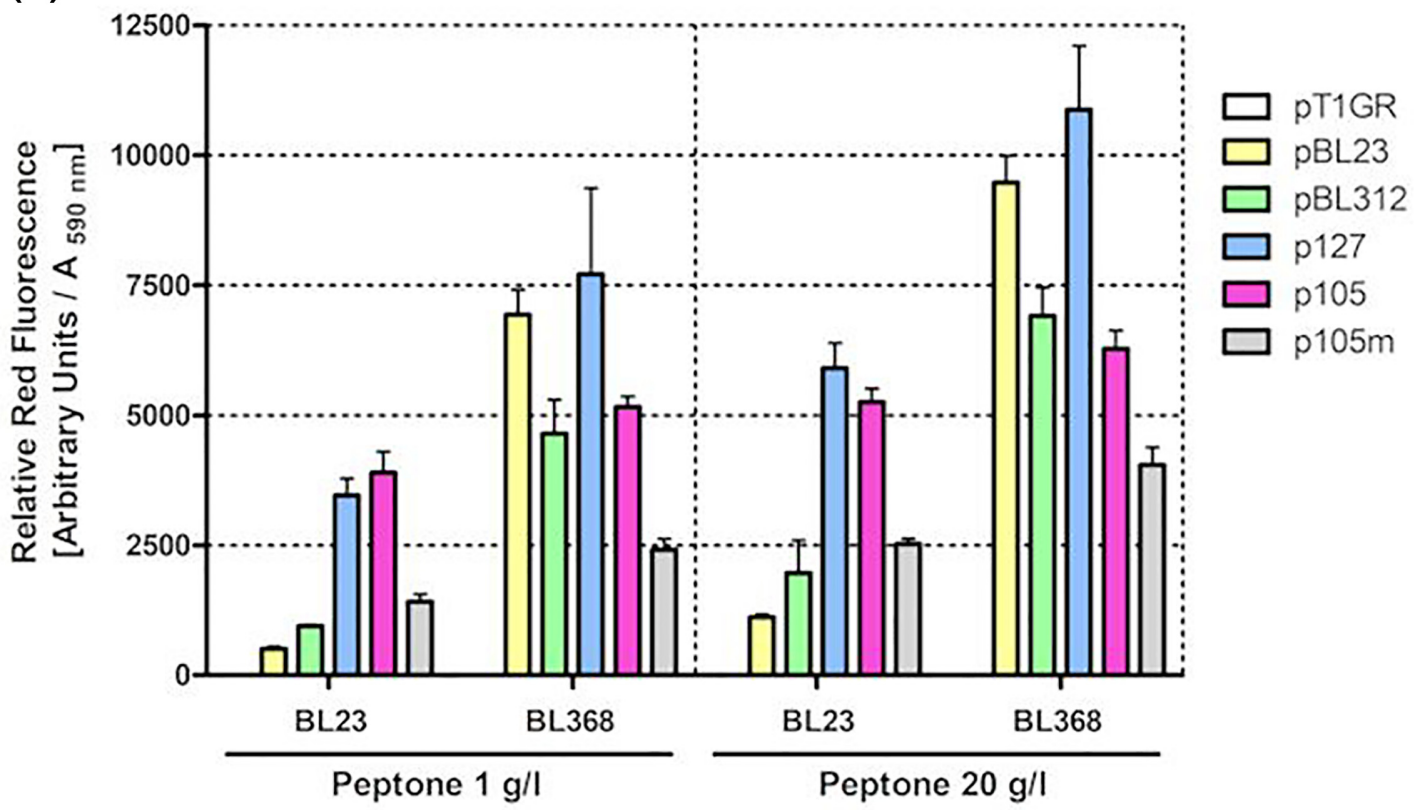

(B)

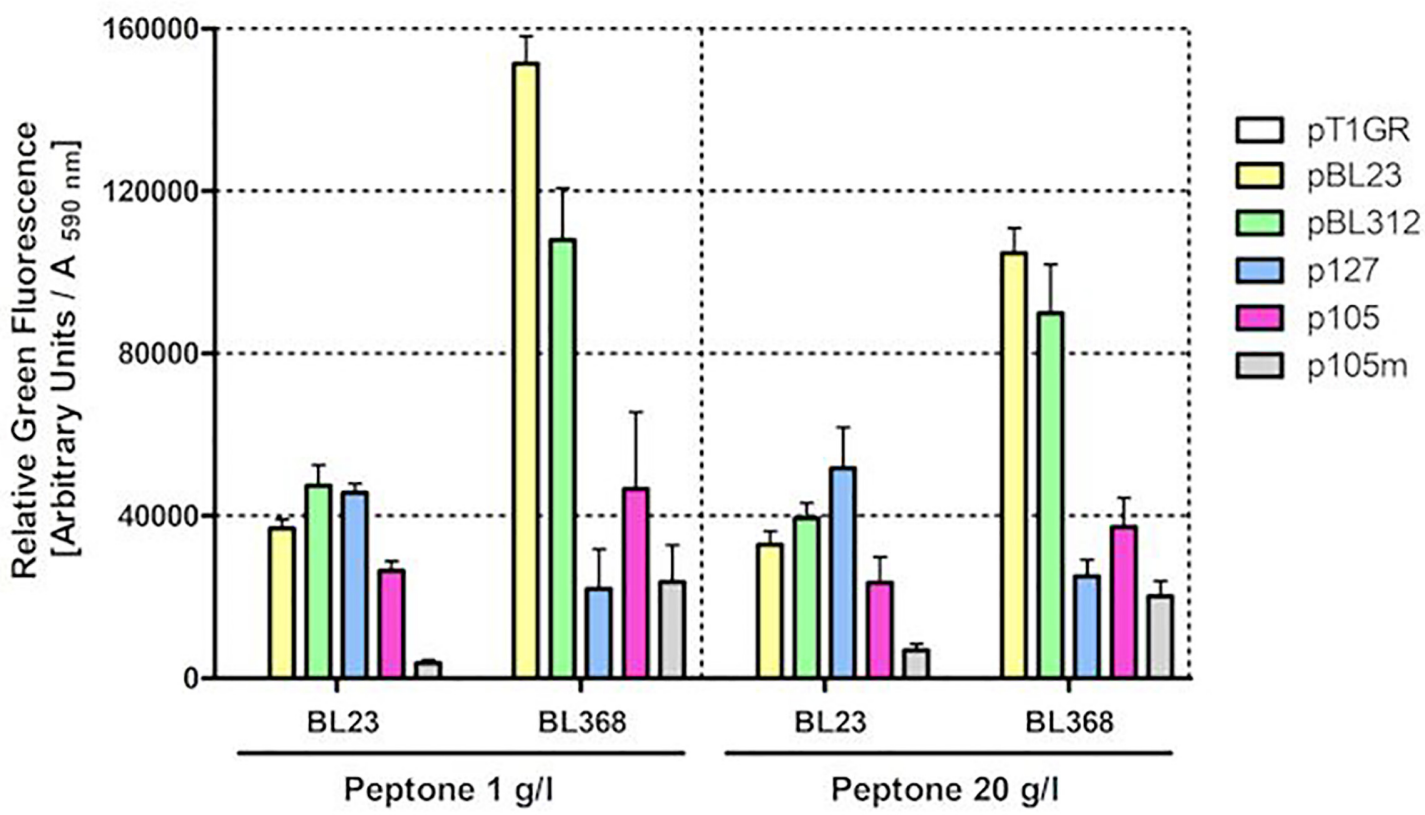

Figure 3. Relative fluorescence generated by the expression of the gene encoding the red fluorescent protein, mRFP, from the PprtP promoter (A) and the green fluorescent protein, GFP, from PprsA promoter in the reporter plasmid pT1GR. Strains L. paracasei BL23 and BL368 were transformed with pT1GR carrying different forms of the prsA-prtP promoter region: pBL23, pBL312, p127, p105 and p105m. For the sake of clarity, only the names of the promoters appear in the abscissa axes. Host strains (BL23 or BL368) are indicated in the histograms over the group of columns corresponding to the different promoters cloned in pT1GR. All strains were grown on SDM supplemented with 1 and 20 g/l peptone. ANOVA showed that for RFP expression (Panel A) in BL23 there was no significant difference between samples, with exception of pBL23-pBL312 and p127-p105, and pBL312-p105 in BL368 (P > 0.01). In case of GFP (Panel B) expression in BL23 revealed some significant difference between pT1GR-p105m and pBL312-p127 ( $\mathrm{p}$ between 0.01-0.05) and in BL368 only p127-p105m were not significantly different $(P>0.01)$. 
transcription of GFP, possibly because of the putative consensus -10 and -35 region identified. Its possible functionality was confirmed by the poorer expression found in p105m (Fig. 2B). All three deleted promoters did not show differences in expression due to the host strain or the presence of peptone.

\section{DISCUSSION}

Probiotic activity relies in a variety of mechanisms of action that are steadily being disclosed. Some of the probiotic mechanisms require the direct interaction of bacteria or bacterial components with the host cells like secreted surface muramidases, cell wall peptidoglycan, surface layer proteins or pili (Fernandez et al. 2011; Sanders et al. 2018), among others. Proteolytic activity of lactobacilli has been shown to specifically hydrolyse allergenic peptides of $\beta$-lactoglobulin (Pescuma et al. 2015) and $\alpha$ S1-caseins (El-Ghaish et al. 2011). Proteinases (CEP) from certain Lactobacillus strains are able to release peptides from caseins inhibiting the angiotensin-converting enzyme (ACE), hence regulating hypertension (Fuglsang et al. 2003). In addition, CEP from L. paracasei VSL\#3 cleaves specifically the proinflammatory chemokine IP10 decreasing inflammatory signals in the mucosa of mice, possibly as a niche survival strategy (von Schillde et al. 2012). In general, CEP in lactic acid bacteria are repressed in the presence of sources of amino acids and short peptides (Hebert, Raya and De Giori 2000; Miladinov, Kuipers and Topisirovi 2001; Pastar et al. 2007; Alcantara et al. 2016). In this work features of the prsAprtP promoter region of two L. paracasei probiotic strains have been compared using red and green fluorescence reporter proteins and quantified expression of prtP, prsA, prtR1 and prtR2 by RT-qPCR. Despite that such proteins may be sensitive to acidic $\mathrm{pH}$, strains and growth conditions were identical, hence results may be useful for comparative purposes. Strains used were the $L$. paracasei isolate from the fully characterized commercial probiotic mixture VSL\#3 (here BL312) (Douillard et al. 2018) and L. paracasei BL23, a strain with probiotic activity, in which two proteins have been characterized with likely antiapoptotic and epithelium repairing properties, CmuA (P40) and CmuB (P75) (Bäuerl et al. 2010). Differences in expression of prtP gene between different strains coincided with previously reported differences in proteinase activities between BL23 and BL312(VSL\#3) (von Schillde et al. 2012) and between BL23 and BL368 (Alcantara et al. 2016). Strains BL368 and BL312(VSL\#3) showed a moderate derepression of prsA, encoding the maturase PrtM. The presence of $20 \mathrm{~g} / \mathrm{L}$ peptone had a prominent effect on the expression of some of the CEP related genes in BL312 and prtP in BL23.

In contrast, cloning of the promoters in plasmid pT1GR did not show evident repression of the reporter RFP expressed from the prtP promoter, as shown by RT-qPCR. PrcR regulates a number of genes in L. paracasei in response to amino acid and peptide sources, but prtP promoter had some particular features (Alcantara et al. 2016) that could help understanding the results of the fluorescent expression vector. In this previous work, Alcantara et al. (2016) showed that this promoter was repressed by peptide sources, but the dephosphorylated form of PrcR could also bind to it and, as consequence, it might bind the prtP promoter in the absence of amino acid sources. This suggested that this promoter region could have a more complex regulation than other promoters regulated by the RR PrcR, possibly affected by the presence of multiple copies of the promoter when present in a plasmid. It is known that multiple copies of a regulated DNA region can titrate interacting elements (Brewster et al. 2014). Here, it was evident that PrcR still binds the promoters born in the plasmid (much higher expression in BL368 than in BL23), but the lack of repression by peptone indicates that there could still be another element that regulate PrcR phosphorylation -or an unknown PrcR effector-, that is titrated out by large numbers of the promoter fragment. This suggested that this promoter region could have a more complex regulation than other promoters regulated by the RR PrcR Considering these special regulatory features, the monitoring of RFP expression suggested that the deleted promoter $p 127$ was partially derepressed because it excluded one of the regulatory elements AAAA recognized by the repressor PrcR in the prsA-prtP promoter region (Fig. 2), yielding a higher expression than the complete fragment ( $p B L 23)$ in the wild type strain BL23. In BL368 -lacking the repressor-, RFP reached its highest level, and pBL23 was fully derepressed, yielding almost as much red fluorescence as $p 127$. The defective 5' region of $p 105$ and $p 105 m$ rendered lack of regulation but poorer expression than $p 127$.

An important aim of this work was to investigate differences between the prsA-prtP promoters of the probiotic strains BL23 and BL312(VSL\#3). They differed in just 7 nucleotide differences and two single base insertion/deletions (Fig. 2) that did not generate significant differences of RFP expression from $p B L 23$ and pBL312 in the wild type strain BL23. In the PrcR mutant (BL368), pBL312 was also derepressed indicating the same type of regulation, but total expression was lower than from $p B L 23$, suggesting that the mutations may indeed affect the efficacy of expression in BL368 that has BL23 genetic background. This may indicate that the higher CEP activity (von Schillde et al. 2012) and mRNA synthesis of prtP gene in BL312 must be due to naturally higher expression levels not only dependent of a PrcR deregulation in this strain, this higher transcription rate was also affecting the other CEP related genes, prsA, prtR1 and prtR2.

Regulation of the PprsA promoter has also been monitored through the expression of GFP and, again, a similar lack of repression by peptone was found, possibly by the same factors that affected PprtP. In BL23, expression of gfp from pBL312 was significantly greater than from its native strain promoter pBL23, also RT-qPCR data showed that BL312(VSL\#3) had higher expression of prsA. In BL368 both native promoters, $p B L 23$ and pBL312, showed a pronounced derepression of GFP expression. The deleted promoters did not contribute much in this part of the study, but for the fact that a new unforeseen promoter 10 and -35 in 127 provided high constitutive expression under both culture conditions and in both strains.

The activity and the role of PrtR paralogs in L. paracasei will require further investigation, but the relative expression of all the genes -within each strain- revealed a generalized low expression profile of prtR1 and prtR2 compared to prtP. The lack of repression of prtR2 rendered apparent high ratio relative to prtP in BL312 on high peptone concentration. Future studies will determine if PrtR1 and PrtR2 are just remains of a common ancestor with Lactobacillus rhamnosus, or if they have a metabolic role.

In conclusion, this study highlighted some features of the prtP-prsA promoters and differences between two probiotic strains in CEP and genes related. The effect of peptone on PrtP expression has been reported in previous studies (Pastar et al. 2007; Alcantara et al. 2016); here promoters from two probiotics (BL23 and BL312(VSL\#3)) showed a remarkable repression of prtP, but only a small effect on prtR1 and prtR2 mRNA synthesis. When increasing the peptone concentration, gene expression in strain BL312(VSL\#3) decreased but remained proportionally higher than BL23, thus indicating that the higher activity/expression of prtP in BL312(VSL\#3) may be related to other strain specific factors, not to the deregulation by 
nitrogen sources. Cloning of the promoters and deleted mutants in an expression vector with divergent fluorescent markers confirmed that the regulation of PprtP-PprsA promoter by PrcR could be complex as suggested before, as PrcR would still bind the promoter under low amino acid supply and high concentration of target DNA.

Understanding the regulation of very relevant functional properties, such as CEP activity, will be essential to control the biological effects, to exploit the potential benefits of these bacteria and for the selection of probiotic strains.

\section{SUPPLEMENTARY DATA}

Supplementary data are available at FEMSLE online.

\section{ACKNOWLEDGMENTS}

Authors wish to thank Dr. P. López and T. Requena for the gift of plasmid pTLGR, Dr. C. de Simone for providing L. paracasei strain from VSL\#3 mix and also acknowledge the Grants of the Spanish Ministry of Science and Universities AGL2010-1567, AGL201570487-P, RTI2018-097982-B-I00, and from the Generalitat Valenciana ACOMP2012/137. We acknowledge support of the publication fee by the CSIC Open Access Publication Support Initiative through its Unit of Information Resources for Research (URICI).

Conflict of interest. This work has no conflict of interests. This research has been funded exclusively with public grants and there was no commercial company funding it.

\section{REFERENCES}

Acedo-Felix E, Perez-Martinez G. Significant differences between Lactobacillus casei subsp. casei ATCC 393T and a commonly used plasmid-cured derivative revealed by a polyphasic study. Int J Syst Evol Microb 2003;53:67-75.

Alcantara C, Coll JM, Blasco A et al. Peptide and amino acid metabolism is controlled by an OmpR-family response regulator in Lactobacillus casei. Mol Microb 2016;100:25-41.

Brewster RC, Weinert FM, Garcia HG et al. The transcription factor titration effect dictates level of gene expression. Cell 2014;156:1312-23.

Broadbent JR, Barnes M, Brennand C et al. Contribution of Lactococcus lactis cell envelope proteinase specificity to peptide accumulation and bitterness in reduced-fat cheddar cheese. Appl Environ Microb 2002;68:1778-85.

Broadbent JR, Strickland M, Weimer BC et al. Peptide accumulation and bitterness in cheddar cheese made using singlestrain Lactococcus lactis starters with distinct proteinase specificities. J Dairy Sci 1998;81:327-37.

Bäuerl C, Perez-Martinez G, Yan F et al. Functional analysis of the p40 and p75 proteins from Lactobacillus casei BL23. J Mol Microb Biot 2010;19:231-41.

Chaves S, Perdigon G, de LeBlanc AdM. Yoghurt consumption regulates the immune cells implicated in acute intestinal inflammation and prevents the recurrence of the inflammatory process in a mouse model. J Food Protection 2011;74: 801-11.

Chen C, Zhao S, Hao G et al. Role of lactic acid bacteria on the yogurt flavour: a review. Int J Food Properties 2017;20:S316-30.

Douillard FP, Mora D, Eijlander RT et al. Comparative genomic analysis of the multispecies probiotic-marketed product VSL\#3. PLoS One 2018;13:e0192452.
El-Ghaish S, Rabesona H, Choiset Y et al. Proteolysis by Lactobacillus fermentum IFO3956 isolated from Egyptian milk products decreases immuno-reactivity of $\alpha$ S1-casein. J Dairy Res 2011;78:203-10.

Fernandez-Espla MD, Garault P, Monnet V et al. Streptococcus thermophilus cell wall-anchored proteinase: release, purification, and biochemical and genetic characterization. Appl Environ Microb 2000;66:4772-8.

Fernandez EM, Valenti V, Rockel C et al. Anti-inflammatory capacity of selected lactobacilli in experimental colitis is driven by NOD2-mediated recognition of a specific peptidoglycan-derived muropeptide. Gut 2011;60:1050-9.

Fuglsang A, Rattray FP, Nilsson D et al. Lactic acid bacteria: inhibition of angiotensin converting enzyme in vitro and in vivo. Antonie Van Leeuwenhoek 2003;83:27-34.

García-Cayuela T, de Cadiñanos LPG, Mohedano ML et al. Fluorescent protein vectors for promoter analysis in lactic acid bacteria and Escherichia coli. Appl Microb Biot 2012;96:171-81.

Gobbato N, Rachid M, Perdigon G. Anti-inflammatory effect of yoghurt in an experimental inflammatory bowel disease in mouse. J Dairy Res 2008;75:497-504.

Hebert EM, Raya RR, De Giori GS. Nutritional requirements and nitrogen-dependent regulation of proteinase activity of Lactobacillus helveticus CRL 1062. Appl Environ Microb 2000;66:5316-21.

Landete JM, Garcia-Haro L, Blasco A et al. Requirement of the Lactobacillus casei MaeKR two-component system for L-malic acid utilization via a malic enzyme pathway. Appl Environ Microb 2010;76:84-95.

Law J, Haandrikman A. Proteolytic enzymes of lactic acid bacteria. Int Dairy J 1997;7:1-11.

Lee M-Y, Ahn K-S, Kwon O-K et al. Anti-inflammatory and antiallergic effects of kefir in a mouse asthma model. Immunobiol 2007;212:647-54.

Miladinov N, Kuipers OP, Topisirovic L. Casitone-mediated expression of the prtP and prtM genes in Lactococcus lactis subsp. lactis BGIS29. Arch Microb 2001;177:54-61.

Morishita T, Deguchi Y, Yajima $\mathrm{M}$ et al. Multiple nutritional requirements of lactobacilli: genetic lesions affecting amino acid biosynthetic pathways. J Bacteriol 1981;148:64-71.

Muñoz-Provencio D, Rodríguez-Díaz J, Collado MC et al. Functional analysis of the Lactobacillus casei BL23 sortases. Appl Environ Microb 2012;78:8684-93.

Pastar I, Begovic J, Lozo J et al. Casitone-dependent transcriptional regulation of the prtP and prtM genes in the natural isolate Lactobacillus paracasei subsp. paracasei. Folia Microb 2007;52:577-84.

Pastar I, Tonic I, Golic N et al. Identification and genetic characterization of a novel proteinase, PrtR, from the human isolate Lactobacillus rhamnosus BGT10. Appl Environ Microb 2003;69:5802-11.

Perez-Arellano I, Zuniga M, Perez-Martinez G. Construction of compatible wide-host range vectors for the cloning of DNA fragments protected by transcriptional terminators. Plasmid 2003;46:106-116.

Pescuma M, Hébert EM, Haertlé T et al. Lactobacillus delbrueckii subsp. bulgaricus CRL 454 cleaves allergenic peptides of $\beta$ lactoglobulin. Food Chem 2015;170:407-14.

Pfaffl MW, Horgan GW, Dempfle L. Relative expression software tool (REST) for group-wise comparison and statistical analysis of relative expression results in real-time PCR. Nucleic Acids Res 2002;30:e36.

Raveschot C, Cudennec B, Coutte F et al. Production of bioactive peptides by Lactobacillus species: from gene to application. Front Microb 2018;9:2354. 
Sanders ME, Benson A, Lebeer S et al. Shared mechanisms among probiotic taxa: implications for general probiotic claims. Curr Op Biot 2018;49:207-16.

Savijoki K, Ingmer H, Varmanen P. Proteolytic systems of lactic acid bacteria. Appl Microb Biot 2006;71:394-406.

Sheikhi A, Banaei H, Yahaghi $\mathrm{N}$ et al. The effect of yogurt probiotic bacteria on pro- and anti-inflammatory cytokine response of mononuclear cells of ulcerative colitis patients. J Clin Immunol 2012;32:394-5.
Siezen RJ. Multi-domain, cell-envelope proteinases of lactic acid bacteria. Antonie Van Leeuwenhoek 1999;76:139-55.

Steidler L, Hans W, Schotte L et al. Treatment of murine colitis by Lactococcus lactis secreting Interleukin-10. Science 2000;289:1352-5.

von Schillde M-A, Hörmannsperger G, Weiher M et al. Lactocepin secreted by Lactobacillus exerts anti-inflammatory effects by selectively degrading proinflammatory chemokines. Cell Host Microbe 2012;11:387-96. 